\title{
Z-boson decays into an invisible dark photon at the LHC, HL-LHC, and future lepton colliders
}

\author{
M. Cobal®, ${ }^{1,2}$ C. De Dominicis $\odot,{ }^{3}$ M. Fabbrichesi, ${ }^{4}$ E. Gabrielli, ${ }^{5,4,6}$ J. Magro, ${ }^{8,2}$ B. Mele $\odot,{ }^{7}$ and G. Panizzo $\odot^{1,2}$ \\ ${ }^{1}$ Dipartimento Politecnico di Ingegneria ed Architettura, Università degli Studi di Udine, \\ via della Scienze 206, 33100 Udine, Italy \\ ${ }^{2}$ INFN, Sezione di Trieste-Gruppo Collegato di Udine, via delle Scienze, 208, 33100 Udine, Italy \\ ${ }^{3}$ Subatech, CNRS/IN2P3, IMT-Atlantique, Université de Nantes, 44307 France \\ ${ }^{4}$ INFN, Sezione di Trieste, via Valerio 2, 34127 Trieste, Italy \\ ${ }^{5}$ Dipartimento di Fisica, Università Degli Studi di Trieste, via Valerio 2, 34127 Trieste, Italy \\ ${ }^{6}$ NICPB, Rävala 10, Tallinn 10143, Estonia \\ ${ }^{7}$ INFN, Sezione di Roma, P.le Aldo Moro 2, 00185 Roma, Italy \\ ${ }^{8}$ Dipartimento di Scienze Matematiche, Informatiche e Fisiche, Università Degli Studi di Udine, \\ via della Scienze 206, 33100 Udine, Italy
}

(Received 2 July 2020; accepted 7 August 2020; published 27 August 2020)

\begin{abstract}
We study the decay of the $Z$ vector boson into a photon and a massless (invisible) dark photon in highenergy collisions. The photon can be used as a trigger for the event, while the dark photon is detected indirectly as missing momentum in the event final state. We investigate the possibility of searching for such a dark photon at the LHC, HL-LHC, and future lepton colliders, and compare the respective sensitivities. As expected, the best result is found for the lepton colliders running at the $Z$ mass, FCC-ee and CEPC, with a final sensitivity to branching ratios of order $O\left(10^{-11}\right)$. We also discuss how to use the photon angular distribution of the events in lepton collisions to discriminate between the dark photon and a pseudoscalar state like the axion.
\end{abstract}

DOI: $10.1103 /$ PhysRevD.102.035027

\section{MOTIVATIONS AND SYNOPSIS}

The two-body Z-boson decay into an isolated photon $\gamma$ and a stable (or metastable) neutral particle beyond the Standard Model (SM), effectively coupled to the $Z$ and $\gamma$ gauge bosons, can give rise to a peculiar experimental signature at high-energy colliders.

The kinematical properties of the detected photon and of the neutral particle, which are both monochromatic in the rest frame of the decaying $Z$, make this process quite attractive in the search for new physics effects-most notably in the case of lepton colliders, for which the monochromatic photon energy is smeared only by bremsstrahlung radiation and detector effects. This feature is lessened at hadron colliders because of the additional challenges in reconstructing the rest frame of the $Z$ boson, due to the characteristic uncertainties in the transverse missing momentum measurement.

Published by the American Physical Society under the terms of the Creative Commons Attribution 4.0 International license. Further distribution of this work must maintain attribution to the author(s) and the published article's title, journal citation, and DOI. Funded by SCOAP ${ }^{3}$.
What are the possible candidates for such a neutral particle?

Within supersymmetric theories, neutral states can be either fermions (neutralinos and gluinos), which, like the SM neutrinos, would only contribute to $Z$ three-body decays, or scalars (sneutrinos), which are too heavy to be produced in the $Z$-boson decay.

Other theoretical frameworks remain open. Here we consider the case of the dark photon, which is a particle belonging to a dark sector-a sector comprising particles interacting only feebly with SM states, and fashioned as a generalization of dark matter.

A dark photon $\bar{\gamma}$ is the gauge boson of a $U(1)_{D}$ group under which dark matter as well as all other dark particles are charged (see e.g., [1] for a recent review). In particular, a massless dark photon [corresponding to an unbroken $U(1)_{D}$ gauge group] does not couple directly to the SM currents, but only through a dipolelike operator of dimension-five [2,3], a distinguishing characteristic that makes the massless case very different from the massive one. ${ }^{1}$

\footnotetext{
${ }^{1}$ For a comprehensive discussion concerning the distinctive features of these two cases, including limits on the possible couplings of the visible photon to dark-sector matter (the socalled millicharged particles), see [1].
} 
As far as its phenomenology is concerned, the massive dark photon interacts (via kinetic mixing effects) like a SM photon. Since the decay of the $Z$ boson into two photons is forbidden by the Landau-Yang theorem [4,5], a $Z$ boson cannot decay into a photon and a massive dark photon via kinetic mixing. On the other hand, the decay of the $Z$ boson into a photon and a massless dark photon is mediated by a one-loop diagram of SM fermions containing the $\bar{\gamma}$ dark dipole operator and the usual electromagnetic vertex for the emission of the SM photon. Then the Landau-Yang theorem does not apply to this case because the interaction vertices of the dark and the ordinary photon are distinguishable. A branching ratio (BR) for $Z \rightarrow \gamma \bar{\gamma}$ between $O\left(10^{-9}\right)$ and $O\left(10^{-11}\right)$ can be expected [6].

In the present $Z$ decay channel, the missing energy could be carried away by neutral bosons other than the dark photon. For example, an axionlike particle (ALP) has been considered and found to have a BR as large as $O\left(10^{-4}\right)$ [7-9]. Also, more exotic cases have been suggested: a Kaluza-Klein graviton in models of large extra dimensions [with a BR around $O\left(10^{-11}\right)$ ] [10] and an unparticle [for which a BR as large as $O\left(10^{-6}\right)$ is possible] [11]. The signature in the latter two cases will be different since the photon is not resonant due to the continuum spectrum of the missing mass. The same signature is shared by the irreducible background $Z \rightarrow \gamma \nu \bar{\nu}$ [12].

At the experimental level, the process

$$
e^{+} e^{-} \rightarrow Z \rightarrow \gamma+X^{0},
$$

where $X^{0}$ stands for undetected neutral particles, was explored at the Large Electron-Positron Collider (LEP), and a limit of $10^{-6}$ at the $95 \%$ CL was found [13] for the corresponding BR when considering a massless $X^{0}$ in the final state.

In this paper, we investigate the possibility of searching for such a massless dark photon in $Z$ decays at the Large Hadron Collider (LHC), the High Luminosity LHC (HL-LHC), and future lepton colliders, and compare the respective sensitivities.

The paper is organized as follows. In Sec. II, we provide the theoretical framework for the effective $Z \gamma \bar{\gamma}$ couplings, and the expression for the corresponding effective Lagrangian. In Sec. III, we study, through Monte Carlo (MC) simulations, the upper limit on the BR of the $Z$ decay into a photon and a dark photon, that can be reached using data collected at the LHC. Performing a simple extrapolation from the result obtained for the LHC, we also estimate the limit on the BR which can be obtained at the HL-LHC. In Sec. IV, we extend the study to future circular colliders, namely, the FCC-ee and the CEPC. At the electron-positron colliders, as expected, the lower level of background compared to hadron colliders and the production of large samples of $Z$ bosons provide the most stringent limit on the BR, with corresponding outstanding discovery potentials. In Sec. V, assuming that the decay has been observed, we look at the angular distribution of the events to establish the spin of the particle carrying away the missing energy (see [14] for a discussion of the spin dependence of the signature), and determine a lower bound on the number of events needed to distinguish the case of the spin-1 dark photon from a spin-0 pseudoscalar. In Sec. VI, we give our conclusions.

\section{THEORETICAL FRAMEWORK}

We consider the effective coupling of the photon $\gamma$ and the $Z$ gauge boson to a massless dark photon $\bar{\gamma}$. The SM fermions couple to a massless dark photon $\bar{\gamma}$ only through radiative corrections induced by loops of dark-sector particles coupling both to the dark photon $\bar{\gamma}$ and to SM fields. The starting point is thus given by considering the leading contribution provided by the magnetic- and electric-dipole operators, described by the effective Lagrangian

$$
\mathcal{L}=\sum_{f} \frac{e_{D}}{2 \Lambda} \bar{\psi}_{f} \sigma_{\mu \nu}\left(d_{M}^{f}+i \gamma_{5} d_{E}^{f}\right) \psi_{f} B^{\mu \nu},
$$

where the sum runs over all SM fields $\psi_{f}, B^{\mu \nu}$ and $e_{D}$ are the $U(1)_{D}$ dark photon field strength and elementary charge (we assume universal couplings), and $\Lambda$ is the effective scale of the dark sector.

The magnetic- $\left(d_{M}^{f}\right)$ and electric-dipole $\left(d_{E}^{f}\right)$ factors can arise, for instance, at one-loop order, in the framework of a UV completion of the dark sector, in which there are messenger fields providing an interaction between SM and dark fields, as discussed, for instance, in [15]. In this case, the scale $\Lambda$ will be associated to the characteristic mass scale of the new physics running in the loop. The scale $\Lambda$ and the couplings $d_{M, E}^{f}$ can be considered as free parameters, since flavor and astrophysics bounds severely constrain only the operator coefficient $d_{M}^{f} / \Lambda^{2}$ for first generation fermions [1].

As shown in [6], a nonvanishing effective coupling $Z \gamma \bar{\gamma}$ can be generated at one loop, inducing the manifestly gauge invariant effective Lagrangian

$$
\mathcal{L}_{\text {eff }}^{(M)}=\frac{e}{\Lambda} \sum_{i=1}^{3} C_{i} \mathcal{O}_{i}(x),
$$

where $e$ is the unit of electric charge, $\Lambda$ is the scale of the new physics, the dimension-six operators $\mathcal{O}_{i}$ are given by

$$
\begin{gathered}
\mathcal{O}_{1}(x)=Z_{\mu \nu} \tilde{B}^{\mu \alpha} A^{\nu}{ }_{\alpha}, \\
\mathcal{O}_{2}(x)=Z_{\mu \nu} B^{\mu \alpha} \tilde{A}_{\alpha}{ }_{\alpha}, \\
\mathcal{O}_{3}(x)=\tilde{Z}_{\mu \nu} B^{\mu \alpha} A^{\nu}{ }_{\alpha},
\end{gathered}
$$

the field strengths $F_{\mu \nu} \equiv \partial_{\mu} F_{\nu}-\partial_{\nu} F_{\mu}$, for $F_{\mu \nu}=(Z, B, A)_{\mu \nu}$, correspond to the $Z$-boson $\left(Z_{\mu}\right)$, dark-photon $\left(B_{\mu}\right)$ and 
photon $\left(A_{\mu}\right)$ fields, respectively, and $\tilde{F}^{\mu \nu} \equiv \varepsilon^{\mu \nu \alpha \beta} F_{\alpha \beta}$ is the dual field strength. The coefficients $C_{i}$ are dimensionless couplings that can be computed from the UV completion of the theory. For the case of the Lagrangian in Eq. (2), they are a function of the couplings $d_{M}^{f}$, the $U(1)_{D}$ unit of charge $e_{D}$, the SM fermion masses, and the $Z$ mass [6].

Analogously, the Lagrangian induced by the electricdipole moment is

$$
\mathcal{L}_{\text {eff }}^{(E)}=\frac{e}{\Lambda} C_{E} \mathcal{O}(x)
$$

where the dimension-six operator is

$$
\mathcal{O}(x)=Z_{\mu \nu} A^{\mu \alpha} B^{\nu}{ }_{\alpha}
$$

and the expression for the coefficient $C_{E}$ for the Lagrangian in Eq. (2) can be found in [6]. The operators in Eqs. (3) and (7) are $C P$ even and odd, respectively.

The amplitudes in momentum space for the decay $Z \rightarrow$ $\gamma \bar{\gamma}$ can be found by taking into account the effective Lagrangians in Eqs. (3) and (7); the total width is given by [6]

$$
\Gamma(Z \rightarrow \gamma \bar{\gamma})=\frac{\alpha M_{Z}^{5}}{6 \Lambda^{4}}\left(\left|C_{M}\right|^{2}+\left|C_{E}\right|^{2}\right)
$$

where $C_{M}=\sum_{i} C_{i}$. Equation (9) is particularly useful when recasting limits on $\Gamma(Z \rightarrow \gamma \bar{\gamma})$ into bounds on the ratios $\left|C_{M, E}\right| / \Lambda^{2}$, valid for the specific model described here.

In the following, we study $\operatorname{BR}(Z \rightarrow \gamma \bar{\gamma})=\Gamma(Z \rightarrow \gamma \bar{\gamma}) /$ $\Gamma_{\text {tot }}^{Z}$ as the observable providing a direct probe to the $Z \rightarrow \gamma \bar{\gamma}$ process, investigating its discovery potential both at present and future hadron and lepton colliders.

\section{HADRON COLLIDERS}

The LHC [16] is a circular superconducting protonsynchrotron situated at the CERN laboratory, which accelerates and collides protons at a center-of-mass (c.m.) energy of $13 \mathrm{TeV}$. LHC hosts two general-purpose experiments which study the collision products: ATLAS [17] and CMS [18]. In this paper we assume an ATLAS-like detector, simulating events at $13 \mathrm{TeV}$ with a total integrated luminosity of $140 \mathrm{pb}^{-1}$, a choice that reproduces conditions similar to those obtained during Run 2 at the LHC. The HL-LHC [19] is the foreseen upgrade of the LHC to achieve instantaneous luminosities a factor of 5 larger than the present LHC nominal value. The upper limit on $\operatorname{BR}(Z \rightarrow \gamma \bar{\gamma})$ at the HL-LHC has been derived from the one obtained for the LHC by taking into account the increase in luminosity [19].

\section{A. The ATLAS detector}

The ATLAS detector, used in the following as a benchmark experimental framework for simulations at hadronic colliders, is a $46 \mathrm{~m}$ long cylinder, with a diameter of $25 \mathrm{~m}$. It consists of six different cylindrical subsystems wrapped concentrically in layers around the collision point to record the trajectories, momenta, and energies of the particles produced in the collision final states. A magnet system bends the paths of the charged particles so that their momenta can be measured. The four major components of the ATLAS detector are the inner detector (ID), the calorimeter, the muon spectrometer and the magnet system. The ID components, embedded in a solenoidal $2 \mathrm{~T}$ magnetic field, cover a pseudorapidity range of $|\eta|<2.5$. The calorimeters cover the range $|\eta|<4.9$, using different techniques suited to the widely varying requirements of the physics processes of interest and of the radiation environment. The total thickness, including $1.3 \lambda$ from the outer support, is $11 \lambda$ at $\eta=0$. In the muon spectrometer, over the range $|\eta|<1.4$, magnetic bending is provided by the large barrel toroid. For $1.6<|\eta|<2.7$, muon tracks are bent by two smaller end-cap magnets. Over the socalled transition region $1.4<|\eta|<1.6$, magnetic deflection is provided by a combination of barrel and end-cap fields [17].

\section{B. Methods}

\section{Monte Carlo simulation}

At the LHC, we study the discovery potential for the decay $Z \rightarrow \gamma \bar{\gamma}$ through the signal process $p p \rightarrow Z \rightarrow \gamma \bar{\gamma}$. The $Z$-boson production fiducial cross section $\sigma_{f i d}$ was calculated at the leading order (LO) in QCD using MadGraph5_aMC@NLO [20] with parton distributions from NNPDF23 [21], summing over all fermionic $p p \rightarrow$ $Z / \gamma^{*} \rightarrow f \bar{f}$ contributions and imposing on the $Z$ decays the same fiducial cuts required on the signal single detected photon, that is, a minimum $p_{T}$ of $10 \mathrm{GeV}$ and $|\eta|<2.5$. The obtained Z-boson production fiducial cross section turns out to be equal to

$$
\sigma_{f i d}=(2.504 \pm 0.006) \times 10^{4} \mathrm{pb}
$$

with an expected number $N_{Z}$ of $Z$ bosons produced at the LHC at its design luminosity of $300 \mathrm{fb}^{-1}$ of $N_{Z}=7.5 \times 10^{9}$. At the HL-LHC, at design luminosity of $3 \mathrm{ab}^{-1}$, the predicted $N_{Z}$ turns out to be $7.5 \times 10^{10}$, that is, 10 times more.

The crucial ingredient in searching proton collisions for the decay $Z \rightarrow \gamma \bar{\gamma}$ is the separation of the signal from the background processes, which is, in general, very challenging.

The three processes taken into account as main background sources are 


$$
\begin{gathered}
p p \rightarrow \gamma+\text { jets, } \\
p p \rightarrow \gamma \nu \bar{\nu}, \\
p p \rightarrow e^{+} \nu_{e} / e^{-} \bar{\nu}_{e} .
\end{gathered}
$$

Regarding process (11a), the unreconstructed energy from jet clustering can mimic missing energy coming from a $\bar{\gamma}$. In process (11b), each neutrino $\nu$ has the same signature of a massless dark photon, appearing as missing momentum in the $Z \rightarrow \gamma+X$ final state. The same holds true for the neutrinos in the process (11c), where electrons are wrongly reconstructed as photons and pass the photon identification requirements [22].

Both signal and background events were generated at the LO using MadGraph5_aMC@NLO, which allows one to compute the full hard process matrix element, including spin correlations. The $Z \gamma \bar{\gamma}$ interaction was modeled by adapting the UFO [23] from [24], which in our notation corresponds to assuming $C_{E}=0$ and $C_{i}=C$ in Eq. (3), by fixing three out of the nine $d_{M}^{f}$ couplings. This allowed us to effectively simulate a simplified version of the full Lagrangian in Eq (3) with one single free parameter $C$, whose value was fixed during simulation to conventionally get $\operatorname{BR}(Z \rightarrow \bar{\gamma} \gamma)=0.5$. These simplifying assumptions in the simulation process do not impact on the simple kinematic distributions studied in the following, leaving open the possibility of recasting the presented exclusion limits on $\operatorname{BR}(Z \rightarrow \bar{\gamma} \gamma)$ into bounds on the overall parameters $C_{M, E}$ of Eq. (9).

Parton shower and hadronization effects were simulated using PYTHIA8 [25,26], while the ATLAS detector response simulation was performed using DELPHES [27]. The simulated processes and the corresponding number of generated events and cross sections are shown in Table I.

In order to optimize the generation step, all samples were produced by requiring a single isolated photon with $p_{T}>$ $10 \mathrm{GeV}$ and a minimum parton transverse momentum $p_{T}>20 \mathrm{GeV}$ in the hard process, where relevant. In the same fashion, three distinct subsamples were simulated for the process $p p \rightarrow \gamma+$ jets, referred to in Table I and in the following as slices: slice I was generated without a cut in the photon energy, slice II required a minimum photon energy $E_{\gamma}^{\min }$ before detector smearing effects ("particle level") of $300 \mathrm{GeV}$, while slice III required a minimum photon transverse momentum $p_{T, \gamma}^{\min }$ of $30 \mathrm{GeV}$. The three slices were eventually merged into a single final sample, properly weighting events in the overlapping regions in such a way as to always obtain the expected yields therein, as given by the corresponding cross section times luminosity.

\section{Event reconstruction}

In order to model a detector as close as possible to ATLAS, the following conditions were specifically implemented. Charged particles were assumed to propagate in a magnetic field of $3.8 \mathrm{~T}$ enclosed in a cylinder of radius $1.29 \mathrm{~m}$ and length $6 \mathrm{~m}$. Photons were reconstructed from clusters of simulated energy deposits in the electromagnetic calorimeter measured in projective towers with no matching tracks. Photons were identified and isolated by requiring the energy deposits in the calorimeters to be within a cone of size

$$
\Delta R=\sqrt{\Delta \eta^{2}+\Delta \phi^{2}}=0.1
$$

around the cluster barycenter. Candidate photons were required to have transverse energy $E_{T}>10 \mathrm{GeV}$, in order to simulate ATLAS photon efficiency calibrations selections [22], and to be within $|\eta| \leq 2.5$.

Electrons and muons were reconstructed from clusters of energy deposits in the electromagnetic calorimeter matched to a track within $\Delta R=0.5$, without any attempt to independently simulate the muon detector response.

Jets were reconstructed with the anti- $k_{T}$ algorithm [28] with a radius parameter $R=0.5$ from clusters of energy deposits at the electromagnetic scale in the calorimeters. This scale reconstructs the energy deposited by electrons and photons correctly but does not include any corrections for the loss of signal for hadrons due to the noncompensating character of the ATLAS calorimeters. A correction to calibrate the jet energy was then applied, such that a sample

TABLE I. Simulated processes with the corresponding number of generated events $\left(N_{\text {sim }}\right)$ and cross sections. The signal cross section is conventionally set following $\operatorname{BR}(Z \rightarrow \gamma \bar{\gamma})=0.5$. Selection cuts for the single hard photon required during generation are also shown, when relevant. The uncertainties quoted on cross sections are purely statistical. For the definition of slices, see text.

\begin{tabular}{lcccc}
\hline \hline Process & Slice & $N_{\text {sim }}$ & $\sigma(\mathrm{pb})$ & Generation cut \\
\hline$p p \rightarrow \gamma \bar{\gamma}$ & $\ldots$ & 150000 & $(2.504 \pm 0.006) \times 10^{4}$ & $\ldots$ \\
$p p \rightarrow \gamma \nu \bar{\nu}$ & $\ldots$ & 150000 & $13.9 \pm 0.2$ & $\ldots$ \\
& I & 14166722 & $(8.31 \pm 0.01) \times 10^{4}$ & $\ldots$ \\
$p p \rightarrow \gamma+$ jets & II & 281057 & $645 \pm 2$ & $E_{\gamma}>300 \mathrm{GeV}$ \\
& III & 3860000 & $(2.468 \pm 0.005) \times 10^{4}$ & $p_{T}^{\gamma}>30 \mathrm{GeV}$ \\
\hline \hline
\end{tabular}


of hadrons of a given energy is reconstructed with that same energy. Candidate jets were required to have $p_{T}>20 \mathrm{GeV}$.

The missing transverse energy $E_{T}^{\text {miss }}$ was computed as the transverse component of the negative vector sum of the momenta of the candidate physics objects.

\section{Event selection}

Only events with at least one reconstructed photon and no jets in the final state were selected, in order to improve discrimination against the main backgrounds. This requirement, together with the loose cuts applied at generation level, will be referred to in the following as "preselection."

In order to maximize the sensitivity of the search, we investigated the possible application of several kinematic cuts, targeting an increase of the signal over square root of background yield ratio $s / \sqrt{b}$ (see Figs. 1-3). The resulting cuts are summarized in Table II, where the invariant transverse mass $M_{T}$ built with the photon and the missing transverse energy is defined through the formula

$$
M_{T}^{2}=2 E_{T}^{\mathrm{miss}} \cdot p_{T}^{\gamma} \cdot\left[1-\cos \Delta \phi\left(\gamma, E_{T}^{\mathrm{miss}}\right)\right],
$$

where $p_{T}^{\gamma}$ is the transverse component of the photon momentum, and $\Delta \phi\left(\gamma, E_{T}^{\text {miss }}\right)$ is the angle between the photon and the missing transverse momenta in the transverse plane. In particular, as shown in Fig. 1, the requirements in $p_{T}^{\gamma}$ and $E_{T}^{\text {miss }}$ target to reject the leading $\gamma+$ jets background, as well as the lower contribution from $\gamma \nu \bar{\nu}$.

In the following, the cuts reported in Table II will be referred to as "selection." The cut efficiencies due to preselection and selection are reported in Table III.

\section{Statistical methods}

We use a simple binned likelihood function $\mathcal{L}(\mu, \boldsymbol{\theta})$ constructed as a product of Poisson probability terms over

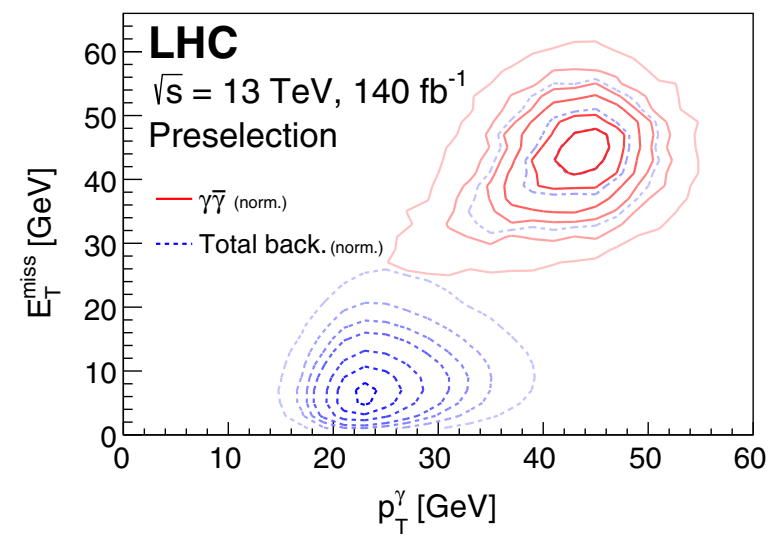

FIG. 1. Normalized distributions in the $p_{T}^{\gamma}-E_{T}^{\text {miss }}$ plane for the signal (red, solid) and total background (blue, dashed) samples, at the preselection level. The contour line color intensity is a linear function of the corresponding normalized distribution values. The low $E_{T}^{\text {miss }}$ region is dominated by $\gamma+$ jets and $\gamma \nu \bar{\nu}$ events.

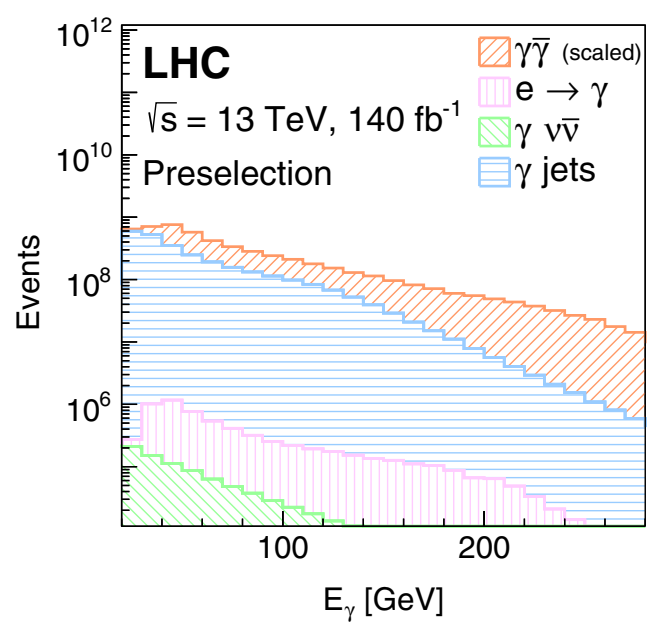

(a)

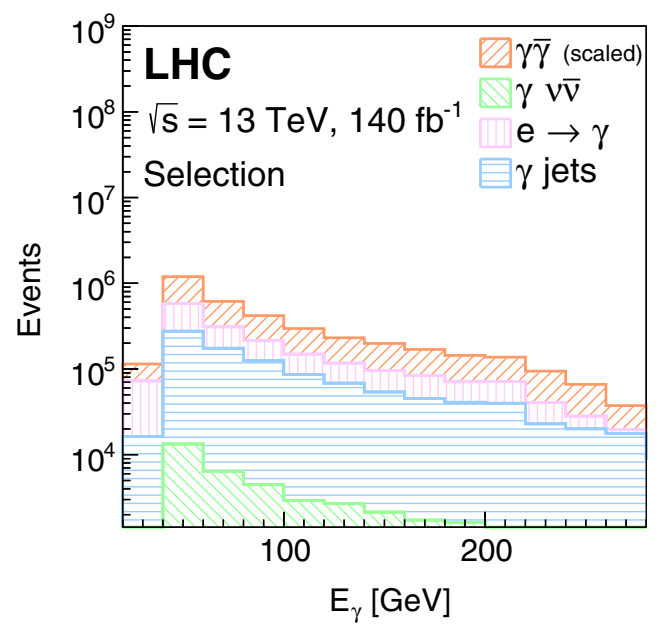

(b)

FIG. 2. Photon energy $E_{\gamma}$ distributions for the signal and background processes, passing (a) preselection and (b) selection requirements. The signal distribution is normalized to the total estimated background yield.

all bins considered [29]. Either $M_{T}$ or $E_{\gamma}$ is used as a discriminating variable, whichever gives the better limits.

The likelihood function is implemented in the ROOSTATS package [30]. It depends on the signal-strength parameter $\mu$, a multiplicative factor that scales the number of expected signal events, and $\boldsymbol{\theta}$, a set of nuisance parameters (NPs) that encode the effect of systematic uncertainties on the background expectations, which are implemented in the likelihood function as Gaussian constraints. One should notice that, in our conventions, we can identify the parameter $\mu$ with the $\operatorname{BR}(Z \rightarrow \gamma \bar{\gamma})$ in the limit $\mu \ll 1$, which always holds throughout the paper when deriving limits. Individual sources of systematic uncertainty are considered to be uncorrelated. The statistical uncertainty of the MC events is not taken into account in $\mathcal{L}(\mu, \boldsymbol{\theta})$ while increasing statistics of MC samples in specific regions of phase space when needed (see e.g. Section III B 1). 


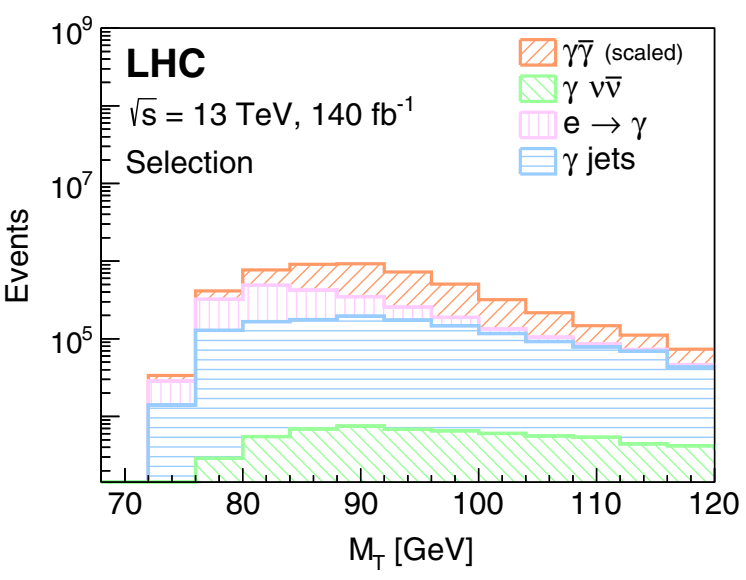

FIG. 3. Transverse mass $M_{T}$ distribution for the signal and background processes after selection cuts in $p_{T}^{\gamma}, E_{T}^{\text {miss }}$ and $\Delta \phi\left(\gamma, E_{T}^{\text {miss }}\right)$. The signal distribution is normalized to the total estimated background yield.

The test statistic $q_{\mu}$ is defined as the profile likelihood ratio

$$
q_{\mu}=-2 \ln \left(\frac{\mathcal{L}\left(\mu, \hat{\hat{\boldsymbol{\theta}}}_{\mu}\right)}{\mathcal{L}(\hat{\mu}, \hat{\boldsymbol{\theta}})}\right),
$$

where $\hat{\mu}$ and $\hat{\boldsymbol{\theta}}$ are the values of the parameters that maximize the likelihood function (with the constraint $0 \leq \hat{\mu} \leq \mu)$, and $\hat{\boldsymbol{\theta}}_{\mu}$ are the values of the NPs that maximize the likelihood function for a given value of $\mu$. The test statistic $q_{\mu}$ is implemented in the ROOFIT package [31]. The expected discovery potential is computed by searching for $\mathrm{BR}(Z \rightarrow \gamma \bar{\gamma})$ values whose excess over SM expectations has a significance of 5 standard deviations. The significance is obtained from the test statistic in the asymptotic limit [32]. Assuming the absence of any significant excess above the background expectation, upper limits on $\mathrm{BR}(Z \rightarrow \gamma \bar{\gamma})$ are derived by using $q_{\mu}$ and the $\mathrm{CL}_{\mathrm{s}}$ method $[33,34]$. Values of $\operatorname{BR}(Z \rightarrow \gamma \bar{\gamma})$ (parametrized by $\mu$ ) yielding $\mathrm{CL}_{\mathrm{s}}<0.05$, where $\mathrm{CL}_{\mathrm{s}}$ is computed using the asymptotic approximation [32], are excluded at 95\% CL.

TABLE II. Cuts applied to maximize $s / \sqrt{b}$. When deriving limits, the cut on $M_{T}$ is applied only when using $E_{\gamma}$ as a discriminating variable (see Sec. III B 4).

\begin{tabular}{ll}
\hline \hline Cut \\
\hline$p_{T}^{\gamma}>35 \mathrm{GeV}$ \\
$E_{T}^{\text {miss }}>40 \mathrm{GeV}$ \\
$\Delta \phi\left(\gamma, E_{T}^{\text {miss }}\right)>2.8 \mathrm{rad}$ \\
$80 \mathrm{GeV}<M_{T}<105 \mathrm{GeV}$ \\
\hline \hline
\end{tabular}

TABLE III. Cut efficiencies after preselection and selection at the LHC for both the signal $\epsilon_{s}$ and the sum of the considered backgrounds $\epsilon_{b}$.

\begin{tabular}{lcc}
\hline \hline Cut & $\epsilon_{s}$ & $\epsilon_{b}$ \\
\hline Preselection & 0.49 & $2.4 \times 10^{-1}$ \\
$p_{T}^{\gamma}$ and $E_{T}^{\text {miss }}$ & 0.27 & $2.3 \times 10^{-4}$ \\
$p_{T}^{\gamma}, E_{T}^{\text {miss }}$ and $M_{T}$ & 0.22 & $1.2 \times 10^{-4}$ \\
$p_{T}^{\gamma}, E_{T}^{\text {miss }}, M_{T}$ and $\Delta \phi\left(\gamma, E_{T}^{\text {miss }}\right)$ & 0.19 & $8.7 \times 10^{-5}$ \\
\hline \hline
\end{tabular}

\section{Results}

We can now derive the upper limit on the $\operatorname{BR}(Z \rightarrow \gamma \bar{\gamma})$ attainable at the LHC.

Let us first look at the best-case scenario in which systematic uncertainties are negligible. We find

$$
\operatorname{BR}(Z \rightarrow \gamma \bar{\gamma})=4 \times 10^{-6}
$$

Though the BR in Eq (15) might compete with the LEP result $\operatorname{BR}(Z \rightarrow \gamma \bar{\gamma})<10^{-6}$ [13] after taking into account the combination of the ATLAS and CMS experiments, or assuming HL-LHC luminosities, this result is weakened by the effect of systematic uncertainties which are unavoidable in the real case.

The level of uncertainty on the background yields will depend on several factors, possibly decreasing with the life of the accelerator due to welcome efforts of the collaborations in understanding and constraining systematic effects. Here an estimate of the overall relative impact $c_{i}=$ $\Delta b_{i} / b$ of the systematic $i$ on the total background yields is attempted, by analyzing a subset of possible systematic uncertainty sources chosen, among the set affecting a search with an analogue signature [35], as the three with highest impact: the uncertainty on $E_{T}^{\text {miss }}$, the jet energy scale uncertainty, and the theoretical uncertainty on the modeling of $\sigma_{b}$. In order to do this, we varied the source of the uncertainty up and down by 1 standard deviation, taken from [35]: we eventually computed the resulting value of $c_{i}$ using the highest variation $\Delta b_{i}=\max \left\{\Delta b_{i}^{\text {up }}, \Delta b_{i}^{\text {down }}\right\}$, as summarized in Table IV. A dedicated uncertainty on $F_{e \rightarrow \gamma}$, the $e \rightarrow \gamma$ fake rate, was assigned to the background from electrons wrongly reconstructed and misidentified as photon candidates [22]. This systematic uncertainty

TABLE IV. Main systematic uncertainty sources on the background yields and corresponding overall relative impact. The sources of systematic uncertainties were taken from [35].

\begin{tabular}{lcc}
\hline \hline Observable & Systematic uncertainty $(\%)$ & $c_{i}$ \\
\hline$E_{T}^{\text {miss }}$ & 1.0 & 0.04 \\
$p_{T}^{\gamma}$ & 0.31 & $<0.01$ \\
$\sigma_{b}$ & 3.7 & 0.04 \\
$F_{e \rightarrow \gamma}$ & 70 & $\cdots$ \\
\hline \hline
\end{tabular}




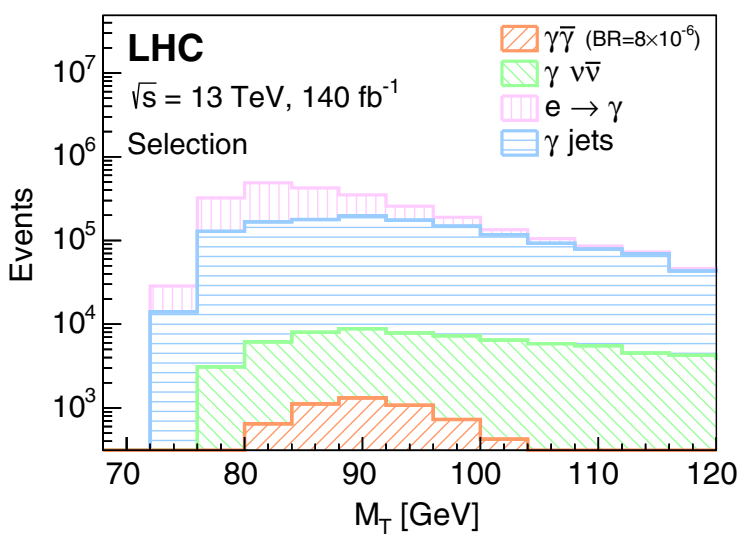

FIG. 4. Transverse mass $M_{T}$ distribution for the signal and background processes after selection cuts in $p_{T}^{\gamma}, E_{T}^{\text {miss }}$ and $\Delta \phi\left(\gamma, E_{T}^{\text {miss }}\right)$ and by scaling the signal distribution by the LHC upper limit $\operatorname{BR}(Z \rightarrow \gamma \bar{\gamma})=8 \times 10^{-6}$.

conservatively covers both converted and unconverted photon fake rates in the central region.

After properly assigning a NP to each source of systematic uncertainty from Table IV in the likelihood function of Sec. III B 4, we compute the 95\% CL upper limit on $\operatorname{BR}(Z \rightarrow \gamma \bar{\gamma})$ considering $L=140 \mathrm{fb}^{-1}$ to be

$$
\operatorname{BR}(Z \rightarrow \gamma \bar{\gamma})<8 \times 10^{-6}
$$

The $M_{T}$ distribution after selection cuts, with signal yields normalized according to Eq. (16), is shown in Fig. 4. This result, compared with Eq. (15), highlights how the study and improvement in the control of systematic uncertainties will be a key feature at the LHC. The corresponding HL-LHC upper limit on $\operatorname{BR}(Z \rightarrow \gamma \bar{\gamma})$ can be estimated under the assumption that the systematic uncertainties will decrease by a factor $1 / \sqrt{L}$. Therefore, the upper limit on $\operatorname{BR}(Z \rightarrow \gamma \bar{\gamma})$ in Eq. (16) can simply be multiplied by a factor $\sqrt{L_{\mathrm{LHC}} / L_{\mathrm{HL}-\mathrm{LHC}}}=\sqrt{140 \mathrm{fb}^{-1} / 3000 \mathrm{fb}^{-1}}$, obtaining

$$
\mathrm{BR}(Z \rightarrow \gamma \bar{\gamma})<2 \times 10^{-6}
$$

which represents the estimate for the best upper limit reachable by a single experiment at the HL-LHC.

From this discussion, it is clear that a search program for the process $p p \rightarrow Z \rightarrow \gamma \bar{\gamma}$ will hardly compete with the LEP result, mainly because of the large background contamination. This negative result is not unexpected, but the exercise of this section is still useful in showing quantitatively the kind of problems one encounters in trying to study this particular process at a hadron collider.

More promising results can be obtained at future lepton colliders, to which we now turn.

\section{FUTURE LEPTON COLLIDERS}

Two circular lepton colliders have currently been proposed. The first one is part of the Future Circular Collider project (FCC), whose integrated program foresees operations in two stages: initially an electron-positron collider (FCC-ee) serving as a Higgs and electroweak factory running at different c.m. energies, followed by a protonproton collider at a collision energy of $100 \mathrm{TeV}$.

The FCC-ee [36] is a high-luminosity, high-precision, $100 \mathrm{~km}$ circumference storage ring collider, designed to provide $e^{+} e^{-}$collisions with center-of-mass energies from 88 to $365 \mathrm{GeV}$. The c.m. operating points with the most physics interest are around $91 \mathrm{GeV}$ (Z-boson pole), $160 \mathrm{GeV}$ ( $W^{ \pm}$pair-production threshold), $240 \mathrm{GeV}$ ( $\mathrm{ZH}$ production), and $340-365 \mathrm{GeV}$ ( $t \bar{t}$ threshold and above). The machine should deliver peak luminosities above $10^{34} \mathrm{~cm}^{-2} \mathrm{~s}^{-1}$ per experiment at the $t \bar{t}$ threshold and the highest ever luminosities at lower energies, with an expected total integrated luminosity $L=150 \mathrm{ab}^{-1}$ at the $Z$ pole.

The other planned electron-positron machine is the Circular Electron Positron Collider (CEPC [37]), which is expected to collide electrons and positrons at different c.m. energies: $91.2 \mathrm{GeV}, 160 \mathrm{GeV}$, and $240 \mathrm{GeV}$ [38]. This machine is expected to provide a total integrated luminosity $L=16 \mathrm{ab}^{-1}$ at the $Z$ pole.

These new lepton colliders will produce huge statistics samples of events (of the order of Tera $Z$ bosons), allowing many measurements with unprecedented accuracy, and the discovery and study of rare $Z, W$, Higgs boson, and top decays. Besides offering the ultimate investigations of electroweak symmetry breaking, these precision measurements will be highly sensitive to the possible existence of yet unknown particles, with masses up to about $100 \mathrm{TeV}$. Sensitive searches for particles with couplings much smaller than weak, such as sterile neutrinos, can be envisioned as well.

We look into the feasibility of a search for a massless dark photon at these new machines, focusing on the centerof-mass energy of $91.2 \mathrm{GeV}$, which represents the most promising setup for the process considered here.

\section{A. FCC-ee detector: IDEA}

The IDEA detector concept [36], developed specifically for FCC-ee, is based on established technologies resulting from years of research and development. However, additional work is needed to finalize and optimize the design.

The detector comprises a silicon pixel vertex detector, a large-volume extremely-light short-drift wire chamber surrounded by a layer of silicon microstrip detectors, a thin, low-mass superconducting solenoid coil, a preshower detector, a dual-readout calorimeter, and muon chambers within the magnet return yoke. Electrons and muons with momenta above $2 \mathrm{GeV}$ and unconverted photons with 
energies above $2 \mathrm{GeV}$ can be identified with efficiencies of nearly $100 \%$ and with negligible backgrounds. The photon energy should be measured to a precision of $11 \% / \sqrt{E} \oplus 1 \%$.

\section{B. The CEPC detector}

Two primary detector concepts have been developed for the construction of the CEPC detector: a baseline, with two approaches for the tracking systems, and an alternative one, with a different strategy for meeting the jet resolution requirements. In the following we briefly describe the baseline approach, which is the one used for simulations.

The baseline detector concept [38] incorporates the particle flow principle with a precision vertex detector, a time projection chamber (TPC) and a silicon tracker, a high granularity calorimetry system, and a 3 Tesla superconducting solenoid followed by a muon detector embedded in a flux return yoke. In addition, five pairs of silicon tracking disks are placed in the forward regions at either side of the interaction point to enlarge the tracking acceptance from $|\cos \theta|<0.99$ to $|\cos \theta|<0.996$.

The performance of the CEPC baseline detector concept has been investigated with a full simulation, as summarized in the following with focus solely on features which have some impact on the study we are presenting here. Electrons and muons with momenta above $2 \mathrm{GeV}$ and unconverted photons with energies above $5 \mathrm{GeV}$ can be identified with efficiencies of nearly $100 \%$ and with negligible backgrounds. The photon energy should be measured to a precision better than $20 \% / \sqrt{E} \oplus 1 \%$. The ionization energy loss $(d E / d x)$ will be measured in the TPC, allowing the identification of low momentum charged particles. Combining the measurements from the silicon tracking system and the TPC, the track momentum resolution will reach $\Delta\left(1 / p_{T}\right) \sim 2 \times 10^{-5} \mathrm{GeV}^{-1}$.

\section{Methods}

\section{Monte Carlo simulation samples}

The fiducial cross section for the SM Z-boson production at lepton colliders with a c.m. energy of $91.2 \mathrm{GeV}$ and, following the same procedure described in Sec. III B 1, with decays within $|\eta|<3$ is

$\sigma\left(e^{+} e^{-} \rightarrow Z / \gamma^{*} \rightarrow f \bar{f}\right)=(6.19 \pm 0.01) \times 10^{4} \mathrm{pb}$.

The signal process $e^{+} e^{-} \rightarrow Z \rightarrow \gamma \bar{\gamma}$ has a distinctive experimental signature. Both the photon and the massless dark photon are monochromatic with an energy of $M_{Z} / 2$ at the $Z$ factory. The massless dark photon has a neutrinolike signature, appearing as missing momentum in the $Z \rightarrow$ $\gamma+X^{0}$ final state, in association with a peak of photon events around the mentioned energy values.

Two main processes can contribute to background events:

$$
\begin{aligned}
& e^{+} e^{-} \rightarrow \gamma \nu \bar{\nu}, \\
& e^{+} e^{-} \rightarrow \gamma e^{+} e^{-} .
\end{aligned}
$$

In process (19a) the photon is the result of initial state radiation by either the electron or the positron, and the $\nu \bar{\nu}$ pair is produced either by the decay of a $Z$ boson produced in the $s$-channel or by $W$-exchange in the $t$-channel. The radiative Bhabha reaction of process (19b) contributes to background events when both the final state electron and positron escape detection. However, the number of background events from this process strongly depends on the geometry of the detector and on the presence of uninstrumented regions, as observed at the LEP [13] and shown in the following sections for the CEPC and FCC-ee detectors.

The hard process, parton-shower, and hadronization steps were simulated closely following the lines of Sec. III B 1. We checked explicitly that relevant kinematic distributions from reconstruction of events with the two different detector configurations of FCC-ee and CEPC closely match in event yields, up to Monte Carlo statistical fluctuations, the minor differences coming only from the slightly different energy resolutions. If not stated explicitly, in the following the configuration for the baseline CEPC detector concept must be understood, with results from the two detectors differing only by the corresponding integrated luminosity.

The simulated processes and the corresponding number of generated events and cross sections assuming collisions between positron and electron beams with $E_{\text {beam }}=$ $45.6 \mathrm{GeV}$ are reported in Table V. For each background process, two slices have been simulated with different $E_{\gamma}^{\min }$ during generation, respectively, of 18 and $30 \mathrm{GeV}$ for the $e^{+} e^{-} \rightarrow \gamma \nu \bar{\nu}$ and $e^{+} e^{-} \rightarrow \gamma e^{+} e^{-}$processes (see Sec. III B 1).

\section{Event reconstruction}

Charged particles were assumed to propagate in a magnetic field of $3.5 \mathrm{~T}$ homogeneously filling a cylindrical region of radius $1.81 \mathrm{~m}$ and length $4.70 \mathrm{~m}$.

TABLE V. Simulated processes with the corresponding number of generated events $\left(N_{\text {sim }}\right)$ and cross sections. As in Table I, the signal cross section is conventionally set following $\operatorname{BR}(Z \rightarrow \gamma \bar{\gamma})=0.5$. See Sec. III B 1 for the definition of the slices I and II.

\begin{tabular}{lcrcc}
\hline \hline Process & Slice & \multicolumn{1}{c}{$N_{\text {sim }}$} & $\sigma(\mathrm{pb})$ & $E_{\gamma}^{\min }(\mathrm{GeV})$ \\
\hline$e^{+} e^{-} \rightarrow \gamma \bar{\gamma}$ & $\ldots$ & 50000 & $(6.19 \pm 0.01) \times 10^{4}$ & $\ldots$ \\
$e^{+} e^{-} \rightarrow \gamma \nu \bar{\nu}$ & I & 5000000 & $5025.0 \pm 4.5$ & $\ldots$ \\
& II & 500000 & $0.1599 \pm 0.0002$ & 18 \\
$e^{+} e^{-} \rightarrow \gamma e^{+} e^{-}$ & I & 5000000 & $8100 \pm 1176$ & $\ldots$ \\
& II & 500000 & $220.9 \pm 0.4$ & 30 \\
\hline \hline
\end{tabular}


Photons were reconstructed from clusters of energy deposits in the electromagnetic calorimeter measured in projective towers with no matching tracks. Photons were identified and isolated by requiring the energy deposits in the calorimeters to be within a cone of size

$$
\Delta R=\sqrt{\Delta \eta^{2}+\Delta \phi^{2}}=0.5
$$

around the cluster barycenter. Candidate photons were required to have $E>2 \mathrm{GeV}$ and to be within $|\cos \theta| \leq 0.987$.

Electrons and muons were reconstructed from clusters in the electromagnetic calorimeter with a matching track. The criteria for their identification were similar to those used for photons.

Jets were reconstructed with the anti- $k_{T}$ algorithm with a radius parameter $R=0.5$ from clusters of energy deposits in the calorimeters (hadronic and electromagnetic). Candidate jets were required to have $p_{T}>20 \mathrm{GeV}$.

The missing energy vector $\vec{p}_{\text {miss }}$ was computed as the negative vector sum of the momenta of the candidate physics objects.

\section{Event selection}

Events were initially selected to have at least one photon and no charged particles in the final state. This requirement, together with the loose cuts applied at generation level, will be referred to in the following as "preselection." Selection cuts were then applied to increase the ratio $s / \sqrt{b}$ and improve the upper limit on the $\operatorname{BR}\left(e^{+} e^{-} \rightarrow \gamma \bar{\gamma}\right)$, as summarized in Table VI.

At leptonic colliders the initial state of the process is fully determined (apart from initial-state-radiation effects) by the beam parameters, contrary to the hadron collider case, and the center-of-mass frame coincides with the laboratory frame. Yet, we find it instructive, in order to better understand the results of Sec. III C, to keep track of the same kinematical observables defined therein. The transverse invariant mass $M_{T}$ in events with one single photon and missing energy simplifies at particle level to the expression

$$
M_{T}=2 p_{T}^{\gamma}
$$

The $M_{T}$ and photon energy $E_{\gamma}$ distributions after selection are shown in Figs. 5 and 6. The preselection and selection

TABLE VI. Preselection and selection efficiencies at the CEPC and FCC-ee detectors at $91.2 \mathrm{GeV}$.

\begin{tabular}{lcc}
\hline \hline Cut & $\epsilon_{s}$ & $\epsilon_{b}$ \\
\hline Preselection & 0.96 & $6.7 \times 10^{-2}$ \\
$\left|\cos \theta_{\gamma}\right|<0.905$ & 0.95 & $3.4 \times 10^{-3}$ \\
$\left|\cos \theta_{\gamma}\right|<0.905$ and $p_{T}^{\gamma}>18 \mathrm{GeV}$ & 0.95 & $2.3 \times 10^{-6}$ \\
\hline \hline
\end{tabular}

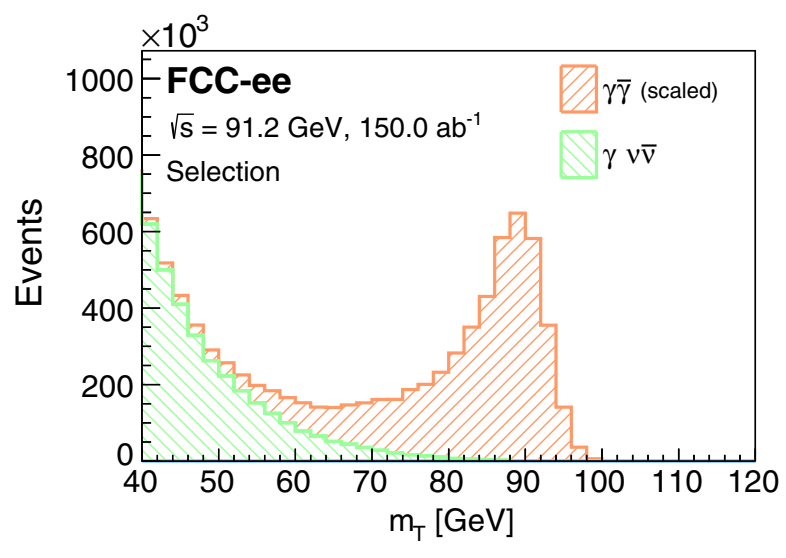

FIG. 5. $M_{T}$ distributions for the signals $e^{+} e^{-} \rightarrow \gamma \bar{\gamma}$ (red) and $e^{+} e^{-} \rightarrow \gamma \nu \bar{\nu}$ (green). No event from the process $e^{+} e^{-} \rightarrow \gamma e^{+} e^{-}$ passed selection requirements. The signal distribution is normalized to the total estimated background yield.

efficiencies are reported in Table VI: no event from the process $e^{+} e^{-} \rightarrow \gamma e^{+} e^{-}$passed selection requirements, as expected from kinematic arguments, thanks to the absence of uninstrumented regions in both the CEPC and FCC-ee detector designs.

\section{Results}

A lepton collider working at a c.m. energy of $91.2 \mathrm{GeV}$ is an actual $Z$ factory and the ideal place to look for the small values of the $\operatorname{BR}(Z \rightarrow \gamma \bar{\gamma})$ we are seeking.

Let us first consider the case with no systematic uncertainties. The lepton colliders perform very well. The CEPC, with a total integrated luminosity $L=16 \mathrm{ab}^{-1}$, yields

$$
\operatorname{BR}(Z \rightarrow \gamma \bar{\gamma})=7 \times 10^{-11} .
$$

The FCC-ee, with an expected total integrated luminosity of $L=150 \mathrm{ab}^{-1}$, gives

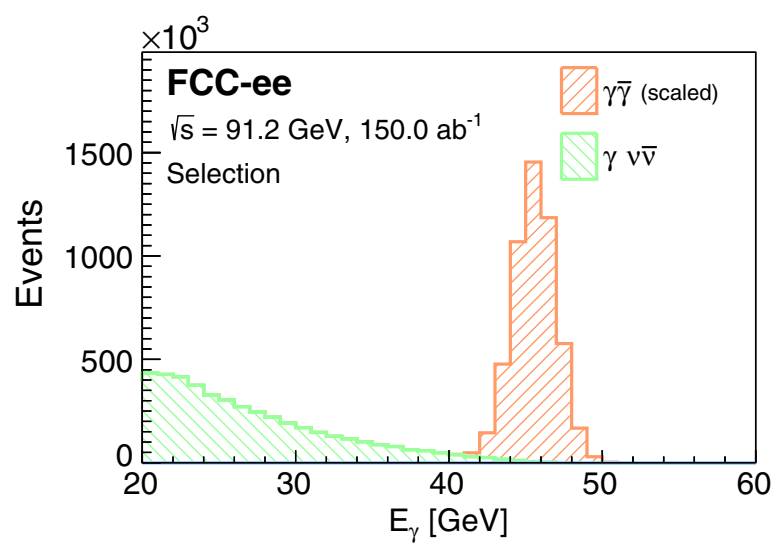

FIG. 6. Photon energy $E_{\gamma}$ distribution after the cut in $\eta$ relative to the signal $\left(e^{+} e^{-} \rightarrow \gamma \bar{\gamma}\right)$ and to the background processes $\left(e^{+} e^{-} \rightarrow \gamma \nu \bar{\nu}\right.$ and $\left.e^{+} e^{-} \rightarrow \gamma e^{+} e^{-}\right)$. The signal distribution is normalized to the total estimated background yield. 
TABLE VII. Summary of the expected 95\% CL upper limits on $\operatorname{BR}(Z \rightarrow \gamma \bar{\gamma})$ obtained from the simulations performed at the LHC, the HL-LHC, and at the FCC-ee and CEPC colliders. The discovery potential is also shown in the last column. Exclusion limits are provided using both $E_{\gamma}$ and $M_{T}$ as discriminating variables, while the discovery potential always uses the better of the two.

\begin{tabular}{lccccc}
\hline \hline & & & \multicolumn{3}{c}{$\operatorname{BR}(Z \rightarrow \gamma \bar{\gamma})$} \\
\cline { 4 - 6 } & $\sqrt{s}$ & $L\left(\mathrm{ab}^{-1}\right)$ & Exclusion $\left(M_{T}\right)$ & Exclusion $\left(E_{\gamma}\right)$ & Discovery \\
\hline LHC & $13 \mathrm{TeV}$ & 0.14 & $8 \times 10^{-6}$ & $5 \times 10^{-5}$ & $2 \times 10^{-5}$ \\
HL-LHC & $13 \mathrm{TeV}$ & 3 & $2 \times 10^{-6}$ & $1 \times 10^{-5}$ & $5 \times 10^{-6}$ \\
FCC-ee & $91.2 \mathrm{GeV}$ & 150 & $2 \times 10^{-11}$ & $3 \times 10^{-11}$ & $6 \times 10^{-11}$ \\
CEPC & $91.2 \mathrm{GeV}$ & 16 & $7 \times 10^{-11}$ & $8 \times 10^{-11}$ & $2 \times 10^{-10}$ \\
\hline \hline
\end{tabular}

$$
\operatorname{BR}(Z \rightarrow \gamma \bar{\gamma})=2 \times 10^{-11}
$$

The results in Eq. (22) and Eq. (23) are not modified by taking into account the uncertainties on both the $\sigma_{Z}^{\text {fid }}$ $\left(0.01 \times 10^{4} \mathrm{pb}\right)$ and the luminosity $\Delta L / L=10^{-4}[38]$. Systematic effects on the upper limit on $\operatorname{BR}\left(e^{+} e^{-} \rightarrow \gamma \bar{\gamma}\right)$ are found to be negligible.

All results of the previous sections are summarized in Table VII. These show that, at both the CEPC and FCC-ee lepton colliders running at the $Z$ pole mass, the limit on the $\mathrm{BR}\left(e^{+} e^{-} \rightarrow \gamma \bar{\gamma}\right)$ will significantly improve the present LEP bound. This motivates the computation of the discovery potential of such lepton colliders, as highlighted in the last column of Table VII: at the CEPC one can discover an excess over SM expectations in $e^{+} e^{-} \rightarrow \gamma \bar{\gamma}$ events with

$$
\operatorname{BR}(Z \rightarrow \gamma \bar{\gamma})=2 \times 10^{-10},
$$

while at the FCC-ee the discovery reach amounts to

$$
\mathrm{BR}(Z \rightarrow \gamma \bar{\gamma})=6 \times 10^{-11} .
$$

These results are quite impressive once compared with the corresponding discovery potential possibly obtained at the LHC and HL-LHC. Finally, expected 95\% CL upper limits for the EFT coefficients of Eq. (9) are summarized in Table VIII.

\section{SPIN ANALYSIS}

Having discussed the discovery potential of a dark photon produced in association with a photon in Z-boson decays, in this last section we investigate how to establish

TABLE VIII. Expected 95\% CL upper limits for the EFT model [6].

\begin{tabular}{lc}
\hline \hline & $\left|C_{M}\right| / \Lambda^{2}\left(\mathrm{TeV}^{-2}\right)$ \\
\hline LHC & 2 \\
HL-LHC & 0.7 \\
FCC-ee & $3 \times 10^{-3}$ \\
CEPC & $5 \times 10^{-3}$ \\
\hline \hline
\end{tabular}

the spin of such a new neutral state. Since nothing prevents the $Z$ boson from decaying into a photon and a pseudoscalar massless neutral particle $a$, the latter can be used as a test hypothesis against the $J^{P}=1^{-}$nature of the dark photon.

No attempt is made here to optimize the search strategy for a pseudoscalar signal $Z \rightarrow a \gamma$, meaning that results presented in previous sections do not necessarily apply to this test hypothesis.

\section{A. Methods}

We now assume that the discovery of $\bar{\gamma}$ in Z-boson decays has occurred at a future $e^{+} e^{-}$collider with $\sqrt{s}=M_{Z}$. In this scenario, a good observable discriminating between the two $J^{P}=1^{-}, 0^{-}$hypotheses is the cosine of the polar angle $\theta$ of the detected photon [14]. The corresponding distributions (Fig. 7) can be produced using the linear realization of the model [39], where the two relevant dimension-five operators regarding $\gamma a$ final states at the $Z$ peak are

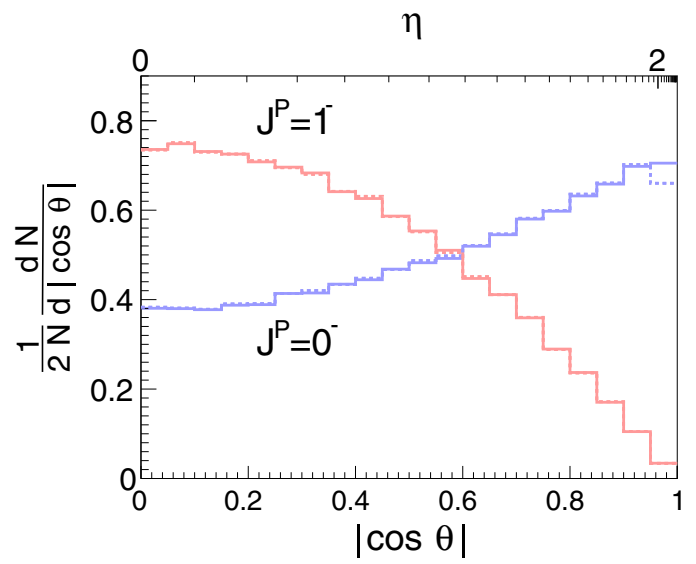

FIG. 7. Differential cross section as a function of the cosine of the detected photon polar angle $\theta$ when produced in association with a pseudoscalar (blue) or vector (red) massless dark particle, after background subtraction. Dashed lines describe the corresponding distribution when including detector smearing effects. The upper axis maps the same range in photon pseudorapidities. 


$$
\begin{aligned}
& \mathcal{O}_{a Z \gamma}(x)=a Z_{\mu \nu} \tilde{A}^{\mu \nu}, \\
& \mathcal{O}_{a \gamma \gamma}(x)=a A_{\mu \nu} \tilde{A}^{\mu \nu},
\end{aligned}
$$

with the latter contributing through interference with the first. We have checked that the independent contributions from $\mathcal{O}_{a Z \gamma}, \mathcal{O}_{a \gamma \gamma}$ and their interference in the $\cos \theta$ distribution are indistinguishable in shape, as expected. One can then apply the following analysis to any combination of the $c_{\tilde{B}}$ and $c_{\tilde{W}}$ couplings and, in particular, for the common and phenomenologically appealing $c_{\tilde{B}}=$ $-\tan \theta_{W} c_{\tilde{B}}$ choice [39], which corresponds to setting $g_{\text {ary }}=0$.

Following the statistical treatment described in Sec. III B 4, a likelihood function $\mathcal{L}\left(J^{P}, \mu, \boldsymbol{\theta}\right)$ that depends on the spin-parity assumption of the signal is constructed as a product of conditional probabilities over the binned distribution of the discriminating observable $\cos \theta$ :

$\mathcal{L}\left(J^{P}, \mu, \boldsymbol{\theta}\right)=\prod_{i}^{N_{\text {bins }}} P\left(N_{i} \mid \mu S_{i}^{\left(J^{P}\right)}(\boldsymbol{\theta})+B_{i}(\boldsymbol{\theta})\right) \times \mathcal{A}(\boldsymbol{\theta})$,

where, given the clean lepton collider environment, a good starting approximation is to assume the best-case scenario in which all nuisance parameters $\boldsymbol{\theta}$ are sufficiently constrained by auxiliary measurements through the functions $\mathcal{A}(\boldsymbol{\theta})$. This is equivalent to making the realistic assumption that other measurements, independent from the one under study, will increase our knowledge about backgroundrelated uncertainties, whose contribution on Eq. (28) can then be safely neglected. In the following we will thus assume that background events can be subtracted from total yields with dedicated methods (see e.g., [40]), with a negligible impact on the discrimination power between the two spin hypotheses.

Closely following [41], a proper test statistic $q$ is then chosen to be the logarithm of the likelihood ratio

$$
q=\log \frac{\mathcal{L}\left(J^{P}=1^{-}, \hat{\hat{\mu}}_{1^{-}}, \hat{\hat{\boldsymbol{\theta}}}_{1^{-}}\right)}{\mathcal{L}\left(J^{P}=0^{-}, \hat{\hat{\mu}}_{0^{-}}, \hat{\boldsymbol{\theta}}_{0^{-}}\right)} .
$$

In this case, given the rather simple form of both Eq. (28) and (29), they have been implemented directly in a ROOT [42] script. The distributions of the test statistic $q$ for both signals shown in Fig. 8 were obtained, as an example, using $n_{\text {toys }}=160000$ pseudo-experiments and with the specific choice of $N=10$ signal events.

The distributions of $q$ are used to determine the corresponding $p_{0}$ values $p_{0}\left(J^{P}=1^{-}\right)$and $p_{0}\left(J^{P}=0^{-}\right)$. For instance, for the tested hypothesis $p_{0}\left(J^{P}=0^{-}\right)$, the expected and observed $p_{0}$ values are obtained by integrating the corresponding test-statistic distribution, respectively, above the $J^{P}=1^{-} q$ distribution median and above the observed value of $q$. The exclusion of the

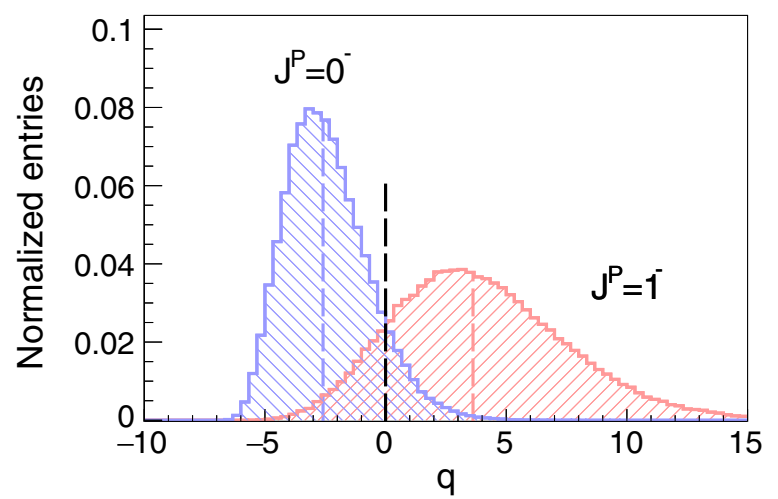

FIG. 8. Expected distributions of the log likelihood ratio test statistics $q$ under the $J^{P}=0^{-}$and $J^{P}=1^{-}$spin hypotheses, both for the $J^{P}=0^{-}$(left) and $J^{P}=1^{-}$(right) signals. Distributions are obtained using $n_{\text {toys }}=160000$ and assuming $N=10$ signal events. The expected medians are indicated by vertical dashed lines, and analogously, the hypothetical observed value is assumed to occur at $q=0$.

$J^{P}=0^{-}$hypothesis in favor of the dark photon $J^{P}=1^{-}$ hypothesis is evaluated in terms of the corresponding $\mathrm{CL}_{\mathrm{s}}\left(0^{-}\right)$, defined as

$$
\mathrm{CL}_{\mathrm{s}}\left(0^{-}\right)=\frac{p_{0}\left(J^{P}=0^{-}\right)}{1-p_{0}\left(J^{P}=1^{-}\right)} .
$$

In the following, we always assume the observed value of the test statistics to be $q=0$.

\section{B. Results}

The example described in Fig. 8 gives an expected $p_{0}\left(J^{P}=0^{-}\right)$value of $3.9 \times 10^{-3}$ and an observed value of $9.3 \times 10^{-2}$. An expected value $p_{0}\left(J^{P}=1^{-}\right)=2.0 \times 10^{-2}$ gives an expected exclusion of the $J^{P}=0^{-}$hypothesis at the $99 \% \mathrm{CL}$, whereas an observed value of $p_{0}\left(J^{P}=1^{-}\right)=$ $1.5 \times 10^{-1}$ gives an observed exclusion at $89 \% \mathrm{CL}$.

Repeating the procedure for all values in the range $N \in[2,25]$, we estimate the lower bound for the expected and observed number of signal events needed to exclude the $p_{0}\left(J^{P}=0^{-}\right)$test hypothesis under the $p_{0}\left(J^{P}=1^{-}\right)$ assumption to be, respectively, $N=6$ and $N=17$ at the $95 \% \mathrm{CL}$.

\section{SUMMARY AND OUTLOOK}

The Z-boson decay into a SM photon and a dark photon would be a most striking signature for the existence of dark photons.

The $Z \rightarrow \gamma \bar{\gamma}$ experimental signature is quite simple and distinctive. In the $Z$-boson c.m. frame, both the photon and the dark photon are monochromatic with an energy of $M_{Z} / 2$. A massless dark photon has a neutrinolike signature in a typical experiment, and it appears as missing momentum in the $Z \rightarrow \gamma+X$ final state. 
In this paper we present the estimate of the best exclusion limits on $\operatorname{BR}(Z \rightarrow \gamma \bar{\gamma})$ for the $Z$ decay into a photon and a dark photon, comparing several present and future collider scenarios: the LHC (at a c.m. energy of $13 \mathrm{TeV}$ with an integrated luminosity of $140 \mathrm{fb}^{-1}$ ), the HL-LHC with $3000 \mathrm{fb}^{-1}$, and the two future circular leptonic machines FCC-ee and CEPC, at the specific design c.m. energy of $91.2 \mathrm{GeV}$ ( $Z$ factory). A summary of the $95 \%$ confidence level upper limits and discovery potentials for the $\operatorname{BR}(Z \rightarrow \gamma \bar{\gamma})$, obtained under the expected conditions of these colliders, is collected in Table VII. It is then straightforward to compare these results with the present LEP bound $\operatorname{BR}(Z \rightarrow \gamma \bar{\gamma})<10^{-6}$ at the $95 \% \mathrm{CL}$.

The impact of systematic uncertainties at the LHC and the challenge of large QCD backgrounds, intrinsic to hadron colliders, make it all but impossible to match the LEP performance. Only a search at the HL-LHC could yield a result that competes with the LEP limit.
At the Z-factories possibly realized at FCC-ee and/or CEPC, instead, limits better than the LEP one could be obtained, thanks to higher luminosities and efficiencies. The much cleaner environment could allow a sensitivity to $\operatorname{BR}(Z \rightarrow \gamma \bar{\gamma})$ of order $O\left(10^{-11}\right)$. Such a value comes close to those predicted in dark-sector models where the effective coupling of the dark photon to the $Z$ boson can be computed.

\section{ACKNOWLEDGMENTS}

M. F. is affiliated with the Physics Department of the University of Trieste and the Scuola Internazionale Superiore di Studi Avanzati (SISSA), Trieste, Italy. M. F. and E. G. are affiliated with the Institute for Fundamental Physics of the Universe (IFPU), Trieste, Italy. The support of these institutions is gratefully acknowledged.
[1] M. Fabbrichesi, E. Gabrielli, and G. Lanfranchi, The dark photon, arXiv:2005.01515.

[2] S. Hoffmann, Paraphotons and axions: Similarities in stellar emission and detection, Phys. Lett. B 193, 117 (1987).

[3] B. A. Dobrescu, Massless Gauge Bosons Other Than the Photon, Phys. Rev. Lett. 94, 151802 (2005).

[4] L. D. Landau, On the angular momentum of a system of two photons, Dokl. Akad. Nauk SSSR 60, 207 (1948).

[5] C. N. Yang, Selection rules for the dematerialization of a particle into two photons, Phys. Rev. 77, 242 (1950).

[6] M. Fabbrichesi, E. Gabrielli, and B. Mele, Z Boson Decay into Light and Darkness, Phys. Rev. Lett. 120, 171803 (2018).

[7] M. Bauer, M. Neubert, and A. Thamm, Collider probes of axion-like particles, J. High Energy Phys. 12 (2017) 044.

[8] J. Kim and U. Lee, $Z^{0}$ decay to photon and variant axion, Phys. Lett. B 233, 496 (1989).

[9] J. A. Dror, R. Lasenby, and M. Pospelov, Dark forces coupled to nonconserved currents, Phys. Rev. D 96, 075036 (2017).

[10] B. C. Allanach, J. P. Skittrall, and K. Sridhar, Z boson decay to photon plus Kaluza-Klein graviton in large extra dimensions, J. High Energy Phys. 11 (2007) 089.

[11] K. Cheung, T. W. Kephart, W.-Y. Keung, and T.-C. Yuan, Decay of $Z$ boson into photon and unparticle, Phys. Lett. B 662, 436 (2008).

[12] J. M. Hernandez, M. A. Perez, G. Tavares-Velasco, and J. J. Toscano, Decay $Z \rightarrow \nu \bar{\nu} \gamma$ in the standard model, Phys. Rev. D 60, 013004 (1999).

[13] P. Abreu et al. (DELPHI Collaboration), Search for new phenomena using single photon events in the delphi detector at lep, Z. Phys. C 74, 577 (1997).

[14] A. Comelato and E. Gabrielli, Untangling the spin of a dark boson in $Z$ decays, Phys. Rev. D 102, 015028 (2020).
[15] E. Gabrielli, L. Marzola, and M. Raidal, Radiative Yukawa couplings in the simplest left-right symmetric model, Phys. Rev. D 95, 035005 (2017).

[16] L. Evans and P. Bryant, LHC machine, J. Instrum. 3, S08001 (2008).

[17] G. Aad et al. (ATLAS Collaboration), The ATLAS experiment at the CERN large hadron collider, J. Instrum. 3, S08003 (2008).

[18] S. Chatrchyan et al. (CMS Collaboration), The CMS experiment at the CERN LHC, J. Instrum. 0803, S08004 (2008).

[19] G. Apollinari, I. B. Alonso, O. Brüning, M. Lamont, and L. Rossi, High-luminosity large hadron collider (HL-LHC): Preliminary design Report, CERN-2015-005, 2015.

[20] J. Alwall, R. Frederix, S. Frixione, V. Hirschi, F. Maltoni, O. Mattelaer, H. S. Shao, T. Stelzer, P. Torrielli, and M. Zaro, The automated computation of tree-level and next-toleading order differential cross sections, and their matching to parton shower simulations, J. High Energy Phys. 07 (2014) 079.

[21] R. D. Ball et al., Parton distributions with LHC data, Nucl. Phys. B867, 244 (2013).

[22] M. Aaboud et al. (ATLAS Collaboration), Measurement of the photon identification efficiencies with the ATLAS detector using LHC Run 2 data collected in 2015 and 2016, Eur. Phys. J. C 79, 205 (2019).

[23] C. Degrande, C. Duhr, B. Fuks, D. Grellscheid, O. Mattelaer, and T. Reiter, UFO-The universal FeynRules output, Comput. Phys. Commun. 183, 1201 (2012).

[24] C. Degrande, N. Greiner, W. Kilian, O. Mattelaer, H. Mebane, T. Stelzer, S. Willenbrock, and C. Zhang, Effective field theory: A modern approach to anomalous couplings, Ann. Phys. (Amsterdam) 335, 21 (2013). 
[25] T. Sjostrand, S. Mrenna, and P.Z. Skands, PYTHIA6.4 physics and manual, J. High Energy Phys. 05 (2006) 026.

[26] T. Sjostrand, S. Ask, J. R. Christiansen, R. Corke, N. Desai, P. Ilten, S. Mrenna, S. Prestel, C. O. Rasmussen, and P. Z. Skands, An introduction to PYTHiA8.2, Comput. Phys. Commun. 191, 159 (2015).

[27] J. de Favereau, C. Delaere, P. Demin, A. Giammanco, V. Lemaître, A. Mertens, and M. Selvaggi (DELPHES 3 Collaboration), DELPHES 3, A modular framework for fast simulation of a generic collider experiment, J. High Energy Phys. 02 (2014) 057.

[28] M. Cacciari, G. P. Salam, and G. Soyez, The anti- $k_{t}$ jet clustering algorithm, J. High Energy Phys. 04 (2008) 063.

[29] ATLAS Collaboration, Reproducing searches for new physics with the ATLAS experiment through publication of full statistical likelihoods, ATL-PHYS-PUB-2019-0292019.

[30] L. Moneta, K. Belasco, K. S. Cranmer, S. Kreiss, A. Lazzaro, D. Piparo, G. Schott, W. Verkerke, and M. Wolf, The RooStats project, Proc. Sci., ACAT2010 (2010) 057 [arXiv:1009.1003].

[31] W. Verkerke and D. P. Kirkby, The RooFit toolkit for data modeling, eConf C0303241, MOLT007 (2003).

[32] G. Cowan, K. Cranmer, E. Gross, and O. Vitells, Asymptotic formulae for likelihood-based tests of new physics, Eur. Phys. J. C 71, 1554 (2011); Erratum, Eur. Phys. J. C73, 2501 (2013).
[33] T. Junk, Confidence level computation for combining searches with small statistics, Nucl. Instrum. Methods Phys. Res., Sect. A 434, 435 (1999).

[34] A. L. Read, Presentation of search results: The CL(s) technique, J. Phys. G 28, 2693 (2002).

[35] M. Aaboud et al. (ATLAS Collaboration), Measurement of the cross section for inclusive isolated-photon production in $p p$ collisions at $\sqrt{s}=13 \mathrm{TeV}$ using the ATLAS detector, Phys. Lett. B 770, 473 (2017).

[36] M. Benedikt, A. Blondel, P. Janot, M. Mangano, and F. Zimmermann (FCC-ee Collaboration), FCC-ee: The lepton collider, Eur. Phys. J. Special Topics 228, 261 (2019).

[37] CEPC Study Group Collaboration, CEPC conceptual design report: Volume 1-Accelerator, arXiv:1809.00285.

[38] M. Dong and G. Li (CEPC Study Group Collaboration), CEPC conceptual design report: Volume 2-Physics \& detector, arXiv:1811.10545.

[39] I. Brivio, M. Gavela, L. Merlo, K. Mimasu, J. No, R. del Rey, and V. Sanz, ALPs effective field theory and collider signatures, Eur. Phys. J. C 77, 572 (2017).

[40] M. Pivk and F. R. Le Diberder, SPlot: A statistical tool to unfold data distributions, Nucl. Instrum. Methods Phys. Res., Sect. A 555, 356 (2005).

[41] G. Aad et al., Evidence for the spin-0 nature of the Higgs boson using ATLAS data, Phys. Lett. B 726, 120 (2013).

[42] R. Brun and F. Rademakers, ROOT: An object oriented data analysis framework, Nucl. Instrum. Methods Phys. Res., Sect. A 389, 81 (1997). 